\title{
Monocyte Chemoattractant Protein-1 Released from Alveolar Macrophages Mediates the Systemic Inflammation of Acute Alveolar Hypoxia
}

\author{
Jie Chao', Paula Donham¹, Nico van Rooijen ${ }^{1}$, John G. Wood ${ }^{1,3}$, and Norberto C. Gonzalez ${ }^{1}$ \\ ${ }^{1}$ Department of Molecular and Integrative Physiology, and ${ }^{2}$ Department of Surgery, University of Kansas Medical Center, Kansas City, Kansas; and \\ ${ }^{3}$ Department of Molecular Cell Biology, Faculty of Medicine, Vrije Universiteit, Amsterdam, The Netherlands
}

\begin{abstract}
Alveolar hypoxia produces rapid systemic inflammation in rats. Several lines of evidence suggest that the inflammation is not initiated by low systemic tissue partial pressure of oxygen $\left(\mathrm{Po}_{2}\right)$ but by a mediator released into the circulation by hypoxic alveolar macrophages. The mediator activates tissue mast cells to initiate inflammation. Monocyte chemoattractant protein-1/Chemokine (C-C motif) ligand 2 (MCP-1/CCL2) is rapidly released by hypoxic alveolar macrophages. This study investigated whether MCP-1 is the mediator of the systemic inflammation of alveolar hypoxia. Experiments in rats and in alveolar macrophages and peritoneal mast cells led to several results. (1) Alveolar hypoxia $\left(10 \% \mathrm{O}_{2}\right.$ breathing, 60 minutes) produced a rapid (5-minute) increase in plasma MCP-1 concentrations in conscious intact rats but not in alveolar macrophage-depleted rats. (2) Degranulation occurred when mast cells were immersed in the plasma of hypoxic intact rats but not in the plasma of alveolar macrophage-depleted rats. (3) MCP-1 added to normoxic rat plasma and the supernatant of normoxic alveolar macrophages produced a concentration-dependent degranulation of immersed mast cells. (4) MCP-1 applied to the mesentery of normoxic intact rats replicated the inflammation of alveolar hypoxia. (5) The CCR2b receptor antagonist RS-102895 prevented the mesenteric inflammation of alveolar hypoxia in intact rats. Additional data suggest that a cofactor constitutively generated in alveolar macrophages and present in normoxic body fluids is necessary for MCP-1 to activate mast cells at biologically relevant concentrations. We conclude that alveolar macrophage-borne MCP-1 is a key agent in the initiation of the systemic inflammation of alveolar hypoxia.
\end{abstract}

Keywords: alveolar macrophages; alveolar hypoxia; systemic inflammation; monocyte chemoattractant protein-1; mast cells

Alveolar hypoxia occurs in a number of clinical settings, and is frequently associated with systemic effects, some of which are thought to include an inflammatory component (1-5). The reduction of inspired partial pressure of oxygen $\left(\mathrm{PO}_{2}\right)$ in rats induces a rapid and widespread inflammatory response in the mesentery $(6)$, skeletal muscle $(7,8)$, and brain $(9)$, as characterized by increased microvascular concentrations of reactive $\mathrm{O}_{2}$ species (10), increased leukocyte endothelial adhesive interactions (6), leukocyte emigration (11), increased vascular permeability as evidenced by the extravasation of albumin in the presence of reduced blood flow (11-13), and the degranu-

\footnotetext{
(Received in original form June 22, 2010 and in final form July 28, 2010)

This work was supported by National Heart, Lung, and Blood Institute grant HL 39443 (N.C.G.). J.C. is a Pre-Doctoral Fellow of the American Heart Association, Midwest Affiliate. P.D. received a Frontiers in Biology Fellowship from the American Physiological Society, Bethesda, Maryland.

Correspondence and requests for reprints should be addressed to Norberto $C$. Gonzalez, M.D., Department of Molecular and Integrative Physiology, University of Kansas Medical Center, Kansas City, KS 66160. E-mail: ngonzale@kumc.edu

This article has an online supplement, which is accessible from this issue's table of contents at www.atsjournals.org

Am J Respir Cell Mol Biol Vol 45. pp 53-61, 2011

Originally Published in Press as DOI: 10.1165/rcmb.2010-02640C on September 2, 2010 Internet address: www.atsjournals.org
}

\section{CLINICAL RELEVANCE}

This research identifies alveolar macrophage-borne monocyte chemoattractant protein-1/chemokine (C-C motif) ligand 2 (MCP-1/CCL2) as the mediator that initiates the systemic inflammation of hypoxia. This study highlights the extrapulmonary effects of alveolar macrophage activation and provides a possible mechanism for the systemic effects seen in some acute pulmonary diseases associated with reduced alveolar partial pressure of oxygen $\left(\mathrm{Po}_{2}\right)$.

lation of perivascular mast cells (MCs) $(7,8,14)$. The degranulation of MCs is a key event: the MC stabilizer cromolyn attenuates all of the markers of inflammation induced by alveolar hypoxia (14). MCs are abundant in the microcirculatory beds in which the inflammatory effects of hypoxia were demonstrated (15-17), and are known to degranulate and release various inflammatory mediators, including leukotriene $\mathrm{B}_{4}$ and platelet activating factor, which were shown to participate in the systemic inflammation of hypoxia (18).

Substantial evidence indicates that the systemic inflammation elicited by alveolar hypoxia is not triggered by a reduction of peripheral tissue $\mathrm{P}_{2}$, but rather by mediators released from alveolar macrophages (AM $\varnothing$ ) and transported by the circulation $(12,16,17)$. The mediator activates local tissue MCs that release inflammatory agents, activating the renin-angiotensin system (13), and initiating systemic inflammation. This sequence of events is supported by several lines of evidence: (1) The selective reduction of cremaster $\mathrm{PO}_{2}$ in intact rats does not produce cremaster MC degranulation and inflammation unless alveolar $\mathrm{PO}_{2}$ is also reduced. In this case, alveolar hypoxia produces cremaster inflammation, even if cremaster microvascular $\mathrm{PO}_{2}$ is normal $(7,8)$. (2) The depletion of $\mathrm{AM} \varnothing$ prevents the increase in leukocyte-endothelial adherence and in vascular permeability produced by alveolar hypoxia in the cremaster microcirculation (12). (3) Plasma obtained from intact hypoxic rats, but not from AM $\varnothing$-depleted hypoxic rats, increases leukocyte-endothelial adherence and vascular permeability in the normoxic cremaster (12). The effect of hypoxic rat plasma is not attributable to inflammatory mediators released into plasma by activated MCs or adherent leukocytes of the donor rat. Furthermore, the agents responsible for inflammation are not generated by blood cells (19). (4) A topical application of supernatant from hypoxic AM $\varnothing$ produces the degranulation of MCs, increased leukocyte-endothelial adherence, and increased vascular permeability in the normoxic cremaster (12). The degranulation of MCs and leukocyte-endothelial adherence also occur in normoxic, mesenteric postcapillary venules after the application of hypoxic AM $\varnothing$ supernatant (16). (5) Primary AM $\varnothing$ cultures undergo a respiratory burst within 15 minutes of reduction of $\mathrm{PO}_{2}$. In contrast, neither peritoneal MCs nor peritoneal macrophage primary cultures are stimulated by hypoxia 
within this time frame (16). However, MCs are rapidly activated when immersed in the supernatants of hypoxic AM $\varnothing$, but not of hypoxic peritoneal macrophages (16).

The nature of the mediators released by $A M \varnothing$ to trigger inflammation is not clear. AM $\varnothing$ are a rich source of oxidants, cytokines, chemokines, growth factors, and arachidonic acid metabolites, which can be secreted in response to several stimuli, including hypoxia $(16,20,21)$. Chemokines are primary products of activated AM $\varnothing(22)$. The chemokine superfamily is subdivided into four subfamilies, which differ with respect to their number and arrangement of conserved cysteine residues at the $\mathrm{N}$ terminus of the primary amino acid sequence (23). Monocyte chemoattractant protein-1 (MCP-1/CCL2), the prototype of the CC-chemokine subfamily, is an attractive candidate as a mediator of the systemic inflammation of alveolar hypoxia. MCP-1 is endowed with chemotactic and activating properties for macrophages $(24), \mathrm{CD}^{+} / \mathrm{CD}^{+} \mathrm{T}$ lymphocytes (25), and MCs, and is critically involved in the regulation of inflammatory processes. MCP-1 induces the degranulation of MCs and leukocyte-endothelial adherence in the rat cremaster microcirculation (26). A possible role of MCP-1 as a putative mediator of the systemic inflammation of alveolar hypoxia was suggested by our previous observation that primary cultures of AM $\varnothing$, but not of peritoneal macrophages or MCs, release MCP-1 within minutes after a reduction of $\mathrm{PO}_{2}$ (16).

The objective of the present study was to provide definitive evidence concerning a possible role of MCP-1 as the circulating mediator that initiates the systemic inflammation of alveolar hypoxia. We reasoned that if MCP-1 does play this role, the plasma concentration of MCP-1 should increase in animals exposed to hypoxia within a time course compatible with systemic inflammation. The magnitude of this increase should be sufficient to elicit systemic effects, and these effects should be clearly attributable to the activation of MCs by AM $\varnothing$-borne MCP-1. The results indicate that MCP-1 is indeed a key initiator of the systemic inflammation of alveolar hypoxia.

\section{MATERIALS AND METHODS}

All procedures were approved by the Animal Care and Use Committee of the University of Kansas Medical Center, an institution accredited by the American Association for the Accreditation of Laboratory Animal Care.

\section{Bronchoalveolar Lavage and Culture of AMØ}

The method of bronchoalveolar lavage (BAL) was described elsewhere (16). Briefly, BAL was performed in male anesthetized SpragueDawley rats (pentobarbital sodium, $40 \mathrm{mg} / \mathrm{kg}$ intraperitoneal). The cells obtained were cultured as described previously (16), and equilibrated with humidified gas mixtures containing $5 \% \mathrm{CO}_{2}$ and $0 \%, 5 \%$, $10 \%$, or $15 \% \mathrm{O}_{2}$ (balance $\mathrm{N}_{2}$ ) for 30 minutes, depending on the protocol. Stable $\mathrm{PO}_{2}$ values were obtained within 2 minutes (16). The supernatant was removed and frozen at $-80^{\circ} \mathrm{C}$ until use, or cultures were used on the same day, depending on the protocol. Cell viability was assessed according to Trypan blue exclusion.

\section{Peritoneal Lavage and Culture of Isolated Peritoneal MCs}

Peritoneal MCs were harvested by lavage of the peritoneal cavity of male anesthetized rats (ketamine $40 \mathrm{mg} / \mathrm{kg}$, atropine $5 \mathrm{mg} / \mathrm{kg}$ intramuscular), as described previously (16). At the end of the experiment, the animals were killed with sodium pentobarbital ( $150 \mathrm{mg} / \mathrm{kg}$ intravenous). MCs were separated from macrophages by differential centrifugation. Purity typically exceeds $95 \%$ (16). MCs were suspended in serum-free Dulbecco's minimum essential medium (DMEM) and used the same day.

\section{Plasma and AMØ Supernatant Filtration}

Samples of plasma from intact and AM $\varnothing$-depleted rats, and from normoxic AM $\varnothing$ supernatants, were filtered using $30 \mathrm{kD}, 50 \mathrm{kD}$, or $100 \mathrm{kD}$ pore size filters and centrifuged at $14,000 \times \mathrm{g}$ for 30 minutes.
The filtrates and the retained fractions were collected and diluted to their original volume with serum-free DMEM. MCP-1 (30 ng/ml) and peritoneal MCs $\left(10^{6}\right.$ cells $\left./ \mathrm{ml}\right)$ were added to the filtrates and retained fractions of each aliquot.

\section{Exposure of Conscious Intact and AMØ-Depleted Rats to Alveolar Hypoxia}

AM $\varnothing$ were depleted by an airway instillation of clodronate-containing liposomes (12). Liposomes were prepared as previously described (27) and were composed of phosphatidylcholine and cholesterol at a 6:1 molar ratio. Clodronate was a gift from Roche Diagnostics (Manheim, Germany). Clodronate-containing or PBS-containing liposomes were administered intratracheally (12). The effectiveness of AM $\varnothing$ depletion was assessed by counting BAL-recovered AM $\varnothing$ at the end of the experiments. Three days after the administration of liposomes, catheters were placed in the carotid artery and jugular vein under pentobarbital anesthesia $(40 \mathrm{mg} / \mathrm{kg}$ intraperitoneal). On the next day, the rats were placed into a Lucite chamber in which $10 \% \mathrm{O}_{2}-90 \% \mathrm{~N}_{2}$ was circulated. Blood samples were obtained before and after 5, 30, and 60 minutes of exposure to hypoxia. Plasma was separated and stored at $-80^{\circ} \mathrm{C}$. Withdrawn blood was replaced by homologous donor blood. The animals were killed with sodium pentobarbital $(150 \mathrm{mg} / \mathrm{kg}$ intravenous). Plasma MCP-1 was measured with a single-analyte ELISA kit (Thermo Scientific, Rockford, IL).

\section{Intravital Microscopy of the Mesenteric Microcirculation}

The procedures have been described in detail previously (10). Briefly, after anesthesia with ketamine $45 \mathrm{mg} / \mathrm{kg}$ and atropine $0.4 \mathrm{mg} / \mathrm{kg}$ intramuscular, the ileocecal portions of the intestines of male Sprague-Dawley rats $(250-300 \mathrm{~g})$ were exteriorized and mounted on a transparent stage. The exposed mesentery was covered with Saran Wrap to minimize gas exchange of the microcirculation with the environment. Single, unbranched postcapillary venules $(20-40 \mu \mathrm{m}$ in diameter) were studied. Leukocyte-endothelial adherence was determined every 5 minutes. The intensity of MC degranulation was determined using image analysis (16) based on the uptake of ruthenium red $(5 \mathrm{mg} / \mathrm{ml})$. At the end of the experiments, the rats were killedwith sodium pentobarbital ( $150 \mathrm{mg} / \mathrm{kg}$ intravenous).

\section{Statistical Analysis}

Data are presented as means \pm SEM. Each preparation served as its own control, with the data obtained after a given treatment compared with the data obtained during the control period. Significance was established using a $t$ test for paired values. Intergroup comparisons were performed using one-way ANOVA, with the Bonferroni correction for multiple comparisons.

\section{RESULTS}

\section{Hypoxia-Induced Release of MCP-1 from Isolated AMØ}

Primary AM $\varnothing$ cultures were equilibrated with gas mixtures selected to reproduce the $\mathrm{PO}_{2}$ at which $\mathrm{AM} \varnothing$ may be exposed in vivo under various conditions. A gas mixture of $15 \% \mathrm{O}_{2}$ provides a $\mathrm{PO}_{2}$ similar to that of normoxic alveolar gas (i.e., $\sim 100 \mathrm{~mm} \mathrm{Hg}$ ). The $10 \%$ and $5 \% \mathrm{O}_{2}$ gas mixtures approximated alveolar $\mathrm{PO}_{2}$ values observed under conditions of moderate $\left(\mathrm{PO}_{2}\right.$ at $\sim 70 \mathrm{~mm} \mathrm{Hg})$ and severe $\left(\mathrm{PO}_{2}\right.$ at $\left.\sim 35 \mathrm{~mm} \mathrm{Hg}\right)$ hypoxia. Equilibration with gas mixtures nominally containing $0 \% \mathrm{O}_{2}$ seldom results in a total absence of $\mathrm{O}_{2}$ in the solution; with the equilibration method used in these experiments, the $\mathrm{PO}_{2}$ of the solution is approximately $5 \mathrm{~mm} \mathrm{Hg}$ (16). Although this is far below the levels to which AM $\varnothing$ may be exposed in vivo, these values approximate the systemic microvascular $\mathrm{Po}_{2}$ values that are observed in rats breathing $10 \% \mathrm{O}_{2}(7,8)$ and to which MCs and systemic tissue macrophages may be exposed during hypoxia.

The basal release of MCP-1 into the supernatant of primary cultures of AM $\varnothing$ equilibrated with $15 \% \mathrm{O}_{2}$ was $200 \pm 32 \mathrm{pg} / 10^{6}$ cells $(N=$ number of cell cultures $=5)$. In contrast, the MCP-1 
released by AM $\varnothing$ during 30 minutes of equilibration with the hypoxic gas mixtures was nearly 20 -fold greater (Table 1 ). However, no correlation was evident between the magnitude of MCP-1 released and the oxygen tension of the equilibrating gas mixtures in the range of $0-10 \% \mathrm{O}_{2}$. The viability of AM $\varnothing$, as assessed by Trypan blue exclusion at the end of the experiments, ranged from $99.4 \% \pm 0.1 \%$ with $15 \% \mathrm{O}_{2}$ to $99.6 \% \pm 0.1 \%$ with $10 \% \mathrm{O}_{2}$.

\section{CCR2b Receptor Antagonist Prevents Degranulation of Isolated MCs Immersed in Supernatant of Hypoxic AM $\varnothing$}

Peritoneal MCs were immersed in the supernatants of AM $\varnothing$ exposed to normoxic $\left(15 \% \mathrm{O}_{2}\right)$ or hypoxic AM $\varnothing\left(5 \% \mathrm{O}_{2}\right)$ gas mixtures. As expected (16), MCs immersed in normoxic AM $\varnothing$ supernatants did not degranulate, whereas hypoxic AM $\varnothing$ supernatants induced a significant degranulation of MCs (Table 2). On the other hand, a significantly lower percentage of peritoneal MCs pretreated with the CCR2b receptor antagonist RS-102895 $(10 \mu \mathrm{M})$ showed degranulation when immersed in supernatants of hypoxic AM $\varnothing$. Pretreatment with RS-102895 did not affect C48/80-induced MC degranulation, which was similar to what occurred with untreated rats (Table 2). Whereas RS102895 blocks the effects of MCP-1 by binding to the specific CCR2b receptor (28), C48/80, a basic MC secretagogue, induces the degranulation of MCs via a receptor-bypassing action that includes the activation of $\mathrm{G}$ proteins (29).

Plasma MCP-1 Concentrations of Intact and of AMØ-Depleted Conscious Rats Breathing $10 \% \mathrm{O}_{2}$

Hypoxia induced a significant increase in plasma MCP-1 concentrations in rats with a normal number of AM $\varnothing$ (Figure $1 \mathrm{~A})$. The mean number of AM $\varnothing$ recovered by BAL in these rats, treated with PBS liposomes, was $7.74 \pm 0.34 \times 10^{6}$ cells $/$ rat $(n=5)$. MCP-1 was already increased after 5 minutes of hypoxia, and remained elevated at similar concentrations for the remainder of hypoxic exposure. On the other hand, for rats in whom AM $\varnothing$ had been depleted with an injection of clodronate liposomes (mean number of AM $\varnothing$ recovered by $\mathrm{BAL}=0.32 \pm 0.05 \times 10^{6}$ cells $\left./ \mathrm{rat} ; n=5\right)$, the plasma MCP-1 concentration remained unchanged from normoxic values for the first 30 minutes, and increased significantly only after 60 minutes of hypoxia, at which time it reached a value of less than $50 \%$ of that seen in rats with a normal AM $\varnothing$ count.

Peritoneal MCs $\left(0.4 \times 10^{6}\right.$ cells $)$ obtained from normoxic rats were immersed in the plasma samples $(0.4 \mathrm{ml})$ obtained from rats before and while breathing $10 \% \mathrm{O}_{2}$. Essentially no degranulation was evident when peritoneal MCs were immersed into plasma samples obtained during the normoxic control period of either intact or AM $\varnothing$-depleted rats (Figure 1B). The degranulation of MCs increased significantly when cells were immersed in plasma obtained when intact rats were breathing $10 \% \mathrm{O}_{2}$. The percentage of degranulated MCs was essentially the same in samples withdrawn at different times of hypoxia. In marked contrast, little degranulation occurred when MCs were immersed in plasma from hypoxic, AM $\varnothing$-depleted rats. This was the case even when MCs were immersed in plasma from the 60-minute hypoxic sample, in which MCP-1 had increased above control levels.

\section{MCP-1 Concentration Dependence of Degranulation of Peritoneal MCs}

The data already described indicate that, in general, degranulation occurred when MCs were immersed in plasma from hypoxic intact rats with high MCP-1 concentrations, and did not occur upon immersion in the plasma of hypoxic АM $\varnothing$ -
TABLE 1. HYPOXIA-INDUCED RELEASE OF MONOCYTE CHEMOATTRACTANT PROTEIN-1 FROM PRIMARY ALVEOLAR MACROPHAGE CULTURES

\begin{tabular}{lcccc}
\hline $\begin{array}{l}\text { Nominal } \\
\text { Percent } \mathrm{O}_{2}\end{array}$ & $15 \%$ & $10 \%$ & $5 \%$ & $0 \%$ \\
\hline$N$ & 5 & 5 & 5 & 5 \\
$\begin{array}{l}\text { Supernatant } \\
\text { concentration } \\
\text { of monocyte } \\
\text { chemoattractant } \\
\text { protein-1 } \\
\text { (pg/10 } / 10^{6} \text { cells) }\end{array}$ & $200 \pm 32$ & $4,051 \pm 591^{*}$ & $3,757 \pm 909^{*}$ & $4,119 \pm 354^{*}$ \\
\hline
\end{tabular}

Values represent mean \pm SE of five primary cultures of alveolar macrophages exposed to each gas mixture.

$N=$ number of primary cultures exposed to each gas mixture.

* $P<0.01$ versus $15 \% \mathrm{O}_{2}$ group.

depleted rats, which showed lower concentrations of MCP-1. To determine whether this was attributable to the difference in MCP-1 concentrations or to other factors associated with AM $\varnothing$ or hypoxia, we studied the dependence of MC degranulation on MCP-1 concentrations. Peritoneal MCs obtained from normoxic intact rats were exposed to increasing concentrations of MCP-1 dissolved in plasma from normoxic intact rats, in supernatants of normoxic AM $\varnothing$, and in normoxic, serum-free DMEM. In all cases, $0.4 \times 10^{6} \mathrm{MCs}$ were immersed in $0.4 \mathrm{ml}$ of solution. The degranulation of MCs was observed 20 minutes after immersion. Figure 2 shows the percentages of degranulated MCs as a function of MCP-1 added to the solution. The data obtained when MCs were immersed in plasma obtained during $10 \% \mathrm{O}_{2}$ breathing (from both intact and AM $\varnothing$-depleted rats; Figure 1B) are included for comparison (Figure 2, open and solid red diamonds). In these samples, the percentage of MC degranulation observed was plotted as a function of the plasma MCP-1 concentration determined by ELISA.

MCs immersed in normoxic rat plasma or normoxic AM $\varnothing$ supernatant showed essentially the same MCP-1 concentrationdependent degranulation of MCs. This relationship also fits the data obtained with MCs immersed in plasma withdrawn from intact rats breathing $10 \% \mathrm{O}_{2}$ (Figure 2, solid red diamonds). MCs immersed in plasma samples obtained during hypoxia in AMØ-depleted rats showed no increase in degranulation (Figure 2, open red diamonds). This was the case even for the sample obtained after 60 minutes of hypoxia. This sample showed an MCP-1 concentration of $8 \pm 1.3 \mathrm{ng} / \mathrm{ml}$, a concentration that should produce substantial degranulation of MCs immersed in normoxic plasma or AM $\varnothing$ supernatant (Figure 2, dotted red vertical line). Taken together, these results show that the plasma concentration of MCP-1 observed in intact hypoxic rats is, by itself, sufficient to induce the degranulation of MCs in vitro, and that the most likely reason for the degranulation of MCs in this case involves the high plasma MCP-1 concentrations observed during alveolar hypoxia. The data also suggest that AM $\varnothing$ are necessary for plasma to elicit the MCP-1mediated degranulation of MCs.

In contrast to normoxic plasma and AM $\varnothing$ supernatants, MCP-1 added to the culture medium failed to increase the degranulation of MCs, even at the highest MCP-1 concentration. To explain this result, we suggest that a substance present in normoxic plasma and in the supernatant of normoxic AM $\varnothing$, but absent in the plasma of AM $\varnothing$-depleted rats, is necessary for $\mathrm{MCP}-1$ to induce the degranulation of MCs in vitro.

\section{Filtration of Plasma and AMØ Supernatant}

To obtain preliminary evidence of a putative cofactor of MCP-1, normoxic plasma from intact and from AM $\varnothing$-depleted rats, as 
TABLE 2. CCRB2 RECEPTOR ANTAGONIST, RS-102895, ATTENUATES DEGRANULATION OF MAST CELLS IMMERSED IN HYPOXIC ALVEOLAR MACROPHAGE SUPERNATANT

\begin{tabular}{lcccccc}
\hline Percentages of & \multicolumn{5}{c}{ Exposed Supernatant of AMO } \\
\cline { 2 - 7 } $\begin{array}{l}\text { Degranulated } \\
\text { Mast Cells }\end{array}$ & $\mathrm{N}$ & $15 \% \mathrm{O}_{2}$ & $\mathrm{~N}$ & $5 \% \mathrm{O}_{2}$ & $\mathrm{~N}$ & $5 \% \mathrm{O}_{2}+\mathrm{C} 48 / 80$ \\
\hline Untreated & 3 & $3.6 \pm 0.5$ & 3 & $74.7 \pm 0.3^{*}$ & 3 & $87.5 \pm 1.7$ \\
RS-102895 & & & 3 & $14.2 \pm 1.3^{* \dagger}$ & 3 & $84.5 \pm 4.3$ \\
\hline
\end{tabular}

Mast cells from the same culture $\left(0.4 \times 10^{6}\right.$ cells $)$ were immersed in supernatants of alveolar macrophages exposed to either $15 \%$ or $5 \% \mathrm{O}_{2}$. The supernatants contained RS-102895, the mast cell secretagogue C48/80, or both. Untreated alveolar macrophage supernatant contained neither of these. Mast cell degranulation was assessed 20 minutes after immersion.

$N=$ number of cell cultures studied.

$+P<0.01$ versus corresponding untreated group.

* $P<0.01$ versus corresponding C48/80 group.

well as the supernatants of normoxic $A M \emptyset$, were filtered through pores of different sizes. MCP-1 was added to the filtrates as well as the retained fractions, to obtain a concentration approximately $30 \mathrm{ng} / \mathrm{ml}$. This concentration produces a substantial degranulation of MCs immersed in the plasma of intact rats or in AMØ supernatants (Figure 2). Peritoneal MCs obtained from normoxic intact rats were then added to the filtrates and retained fractions, and the degranulation of MCs was determined 20 minutes later. The results obtained using either the plasma from intact rats or AM $\varnothing$ supernatants were essentially the same (Table 3). On the other hand, no degranulation occurred when MCs were added to any of the filtrates or the retained fractions of plasma obtained from AM $\varnothing$-depleted rats.

MCP-1 induced the degranulation of MCs in only two cases: in the fraction of plasma or AM $\varnothing$ supernatant retained by the $30-\mathrm{kD}$ filter, and in the filtrate of the $100-\mathrm{kD}$ filter. No degranulation of MCs was evident in either fraction when the 50-kD filter was used. The best explanation for these results contends that the MCP-1 cofactor is composed of two elements: one with a size between $30-50 \mathrm{kD}$, and another with a size larger than $50 \mathrm{kD}$ and smaller than $100 \mathrm{kD}$. Both elements would be together in the retained fraction of the $30-\mathrm{kD}$ filter, as well as in the filtrate of the $100-\mathrm{kD}$ filter. The degranulation of MCs in either fraction of the 50-kD filter would not occur because the cofactor components are separated by the filter.

In addition to providing evidence for the existence of this cofactor, the data also suggest that the cofactor originates in AMØ. Similar results were obtained by immersing MCs into plasma or AM $\varnothing$ supernatants, suggesting that this substance is released by AM $\varnothing$ into the plasma. This idea is supported by the finding that no evidence exists for the presence of a cofactor in the plasma of AM $\varnothing$-depleted rats. These data are consistent with the observation that MCs did not degranulate when immersed in the plasma sample obtained after 60 minutes of hypoxia in AM $\varnothing$-depleted rats, which showed a significant increase in MCP-1 concentrations (Figure 2).

\section{CCR2b Receptor Antagonist Prevents the Increase in Inflammatory Markers Produced by Alveolar Hypoxia in the Mesentery of Intact Rats}

These experiments were performed to explore the possible role of MCP-1 in the systemic inflammation of alveolar hypoxia in anesthetized, intact rats. Figure 3 shows representative microphotographs of a mesenteric postcapillary venule of an intact rat obtained before (left) and after 30 minutes (center) of $10 \% \mathrm{O}_{2}$ breathing. The microphotograph (right) shows the effects of 30 minutes of alveolar hypoxia in a rat pretreated with RS$102895(10 \mu \mathrm{M}$, applied topically). Hypoxia in untreated rats
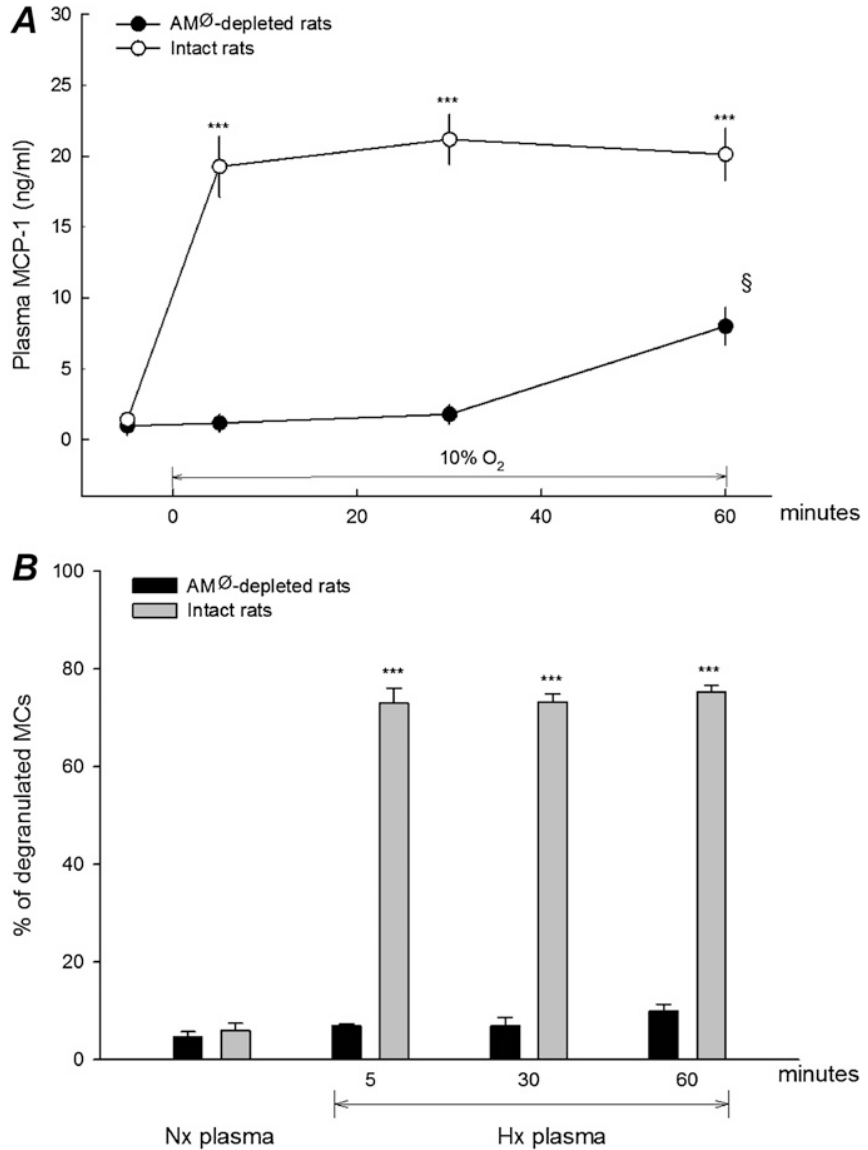

Figure 1. (A) Plasma monocyte chemoattractant protein-1 (MCP-1) concentrations in conscious alveolar macrophage (AMØ)-depleted (solid circles) and intact (open circles) rats before and during 1 hour of breathing $10 \% \mathrm{O}_{2}$. AM $\varnothing$ were depleted by tracheal instillation of clodronatecontaining liposomes 4 days before the experiment. Intact rats received PBS-containing liposomes. Bars are $1 \mathrm{SE}$ on either side of the mean, $n=5$ rats per group for each data point. ${ }^{* *} p<0.01$ versus corresponding normoxic control rats. ${ }^{\S} P<0.01$ versus corresponding value in intact rats. (B) Percentage of degranulated mast cells (MCs) after immersion in rat plasma. Peritoneal MCs $\left(0.4 \times 10^{6}\right.$ cells $)$ from normoxic, intact rats were immersed in $0.4 \mathrm{ml}$ of plasma obtained from rats depicted in $A$ at times indicated in horizontal axis. Solid bars, AMØ-depleted rats; gray bars, intact rats. ${ }^{* * *} P<0.01$ versus corresponding normoxic control rats. $\S P<0.01$ versus corresponding value in intact rats.

resulted in the expected increases of inflammatory markers, according to the MC degranulation index and leukocyteendothelial adherence. These effects of alveolar hypoxia were absent in rats pretreated with RS-102895.

\section{Effects of Topical MCP-1 on the Microcirculation of Intact and AMø-Depleted Rats}

The data in Figure 3 suggest that MCP-1 participates in the mesenteric degranulation of MCs and the increased leukocyteendothelial adherence that occur during the onset of alveolar hypoxia. Further evidence to this effect was obtained using an alternative approach, namely, determining the effects of the local administration of MCP-1 on normoxic rats with a normal AM $\varnothing$ count.

The topical application of MCP-1 (30 ng/ml dissolved in serum-free DMEM) to the mesentery of intact, normoxic rats mimicked the response to alveolar hypoxia (compare center panels of Figures 3 and 4). The pretreatment of the mesentery 
with RS-102895 prevented the response to MCP-1 (Figure 4, right).

In contrast, the effects of MCP-1 on the mesentery of AM $\varnothing$ depleted rats depended on the vehicle into which the MCP-1 had been dissolved. MCP-1 at $30 \mathrm{ng} / \mathrm{ml}$, dissolved in serum-free DMEM, had no effect in the mesentery of AM $\varnothing$-depleted rats (Figure 5, left). On the other hand, the topical application of equal amounts of MCP-1 dissolved in plasma from normoxic rats with a normal AM $\varnothing$ count produced an increase in inflammatory markers (Figure 5, right) The effects of MCP-1 here were similar to those seen when MCP-1 dissolved in serum-free DMEM was applied to intact rats (Figure 4, center).

\section{DISCUSSION}

The present experiments demonstrate that MCP-1 plays a central role in initiating the systemic inflammatory response to alveolar hypoxia. The results also show that a cofactor, normally present in normoxic plasma and originating in AM $\varnothing$, is necessary for the stimulatory effect of MCP-1 on MCs.

Our previous work provided compelling evidence that the systemic inflammation of hypoxia is not initiated by reduced tissue $\mathrm{PO}_{2}$, but rather by a mediator released from AM $\varnothing$ and transported by the circulation $(7,8,12,13,16,19)$. A role for AMØ-borne MCP-1 as the putative mediator was suggested by the recent finding that primary cultures of $\mathrm{AM} \varnothing$, but not of peritoneal MCs or peritoneal macrophages, release MCP-1 within 30 minutes of exposure to hypoxia (16). Of 12 different chemokines and cytokines investigated, MCP-1 was the only MC secretagogue released by AM $\varnothing$ within a time frame compatible with a role as a trigger of inflammation. The hypoxia-induced release by AM $\varnothing$ of a MC secretagogue such as MCP-1 is consistent with the observation that cultures of hypoxic AM $\varnothing$, but not of hypoxic peritoneal macrophages, elicited the degranulation of immersed MCs and induced inflammation in tissues of normoxic animals $(12,16)$. Although these data provide evidence for the participation of MCP-1 in the inflammatory process, several criteria must be met to demonstrate that MCP-1 is the AM $\varnothing$-borne circulating agent that initiates the systemic inflammatory cascade. First, a reduction of alveolar $\mathrm{PO}_{2}$ in intact animals must produce an increase in plasma $\mathrm{MCP}-1$ concentration, and this increase must follow a pattern consistent with the time course of the inflammatory response. Second, AM $\varnothing$ must be identified as the source of the elevated plasma MCP-1 concentration in intact animals. Third, the plasma concentration of MCP-1 observed in hypoxic animals must be sufficient to elicit the activation of MCs, the target of the putative circulating mediator. Finally, evidence must show that MCP-1 is directly involved in the

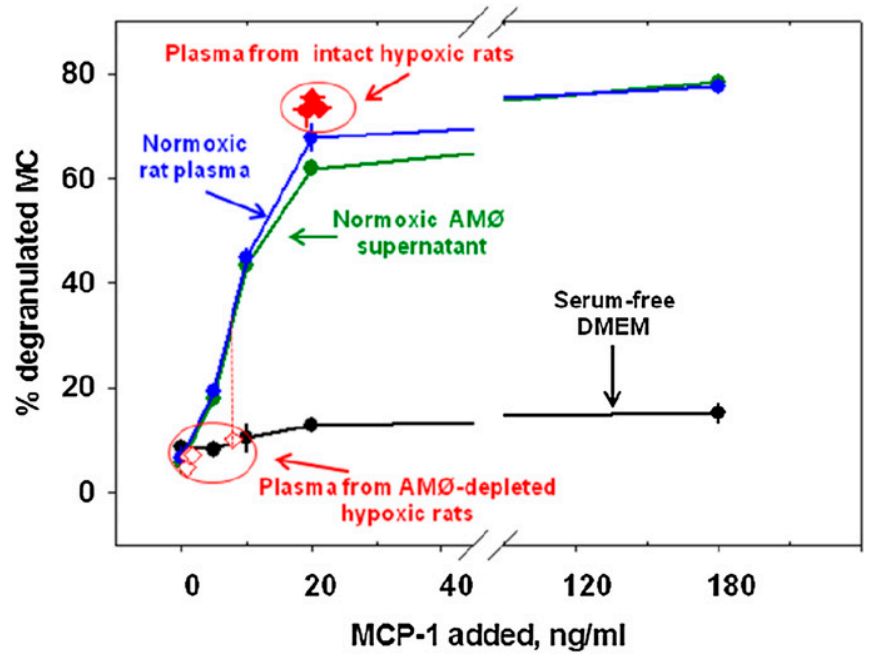

Figure 2. Peritoneal MCs from normoxic, intact rats $\left(0.4 \times 10^{6}\right.$ cells $)$ were immersed in $0.4 \mathrm{ml}$ of solutions containing increasing concentrations of MCP-1. MCs were immersed in normoxic AM $\varnothing$ supernatant (green), in normoxic plasma (blue), and in serum-free Dulbecco's minimum essential medium (DMEM) culture medium (black). Data represent means \pm SE of three experiments per group for each data point. Red diamonds represent data obtained by immersing MCs in plasma drawn from AM $\varnothing$-depleted rats (open diamonds) or intact rats (solid diamonds) breathing $10 \% \mathrm{O}_{2}$, as depicted in Figure 1 .

systemic inflammation of hypoxia in intact animals. According to the data presented here, all these criteria were met to identify MCP-1 as the agent that initiates systemic inflammation. In addition, the results indicate that a cofactor in normoxic biological fluids is necessary for MCP-1 to activate mast cells. The evidence supporting a role for MCP-1 as the key initiator of inflammation will be discussed, followed by a discussion of the data that point to the existence of a cofactor for MCP-1.

\section{AMØ-Borne MCP-1 Initiates the Systemic Inflammation of Hypoxia}

Breathing $10 \% \quad \mathrm{O}_{2}$ elicited a rapid and sustained increase in plasma MCP-1 concentration in intact, conscious rats (Figure 1A). Within 5 minutes, MCP-1 had reached levels that were maintained for 1 hour of hypoxia. This rapid increase matched the time course of the degranulation of MCs and of the leukocyte-endothelial adherence observed in the mesenteric and cremaster microcirculations of rats exposed to alveolar hypoxia $(6,8,14)$.

TABLE 3. EFFECTS OF DIFFERENT FILTERED FRACTIONS OF NORMOXIC PLASMA AND ALVEOLAR MACROPHAGE SUPERNATANT ON DEGRANULATION OF IMMERSED MAST CELLS

\begin{tabular}{|c|c|c|c|c|}
\hline \multirow[b]{2}{*}{ Pore Size } & \multirow[b]{2}{*}{ Fraction } & \multicolumn{3}{|c|}{ Percentages of Degranulated Mast Cells } \\
\hline & & $\begin{array}{l}\text { Plasma of Intact } \\
\text { Rats }(n=3)\end{array}$ & $\begin{array}{c}\text { Supernatant of } \\
\text { Normoxic Alveolar Macrophages }(n=3)\end{array}$ & $\begin{array}{c}\text { Plasma of Alveolar } \\
\text { Macrophage-Depleted Rats }(n=3)\end{array}$ \\
\hline \multirow[t]{2}{*}{$30 \mathrm{kD}$} & Retained & $76.7 \pm 1.3^{*}$ & $72.6 \pm 1.6^{*}$ & $5.4 \pm 1.1$ \\
\hline & Filtrate & $5.2 \pm 1.3$ & $4.8 \pm 1.5$ & $6.2 \pm 1.3$ \\
\hline \multirow[t]{2}{*}{$50 \mathrm{kD}$} & Retained & $5.4 \pm 2.6$ & $4.3 \pm 0.9$ & $6.0 \pm 1.4$ \\
\hline & Filtrate & $5.1 \pm 0.4$ & $5.6 \pm 1.3$ & $6.7 \pm 0.2$ \\
\hline \multirow[t]{2}{*}{$100 \mathrm{kD}$} & Retained & $3.7 \pm 1.5^{\star}$ & $5.4 \pm 1.0^{*}$ & $6.8 \pm 0.8$ \\
\hline & Filtrate & $75.0 \pm 3.4$ & $75.2 \pm 1.2$ & $6.1 \pm 1.0$ \\
\hline
\end{tabular}

Monocyte chemoattractant protein-1 $(30 \mathrm{ng} / \mathrm{ml})$ and mast cells $\left(0.4 \times 10^{6}\right.$ cells in a final volume of $\left.0.4 \mathrm{ml}\right)$ were added to the retained fraction or the filtrate.

Degranulation of mast cells was assessed 20 minutes after immersion.

$n=$ number of primary cultures.

* $P<0.01$ versus corresponding filtrate. 


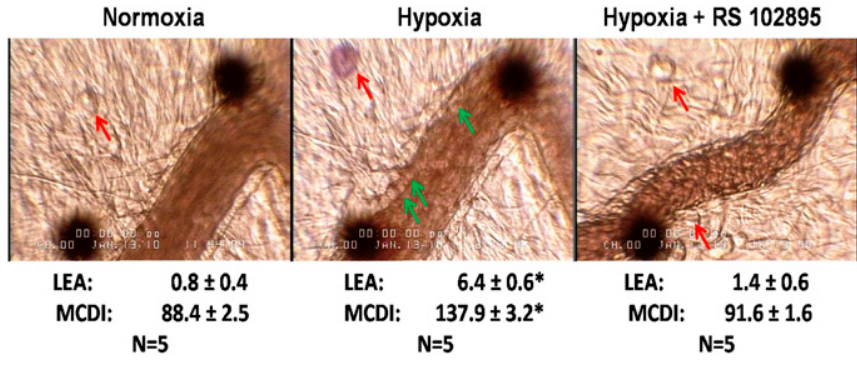

Figure 3. Representative microphotographs of postcapillary mesenteric venules of intact rats. Large black dots are used to align the optical Doppler velocimeter used to measure red cell velocity, and are occasionally moved from the vessel center to obtain a better view of the leukocyte-endothelial interface for photographs. Left and center: Photographs were taken before and after 30 minutes of breathing $10 \%$ $\mathrm{O}_{2}$, respectively. Red arrows indicate MCs, and green arrows indicate adherent leukocytes. Hypoxia induces the degranulation of MCs, as shown by the uptake of ruthenium red, and increased adherence of leukocytes to the endothelium. Right: Venule of an intact rat pretreated with the CCR2b receptor antagonist RS-102895 (10 $\mu \mathrm{M}$, applied topically). This photograph was obtained at 30 minutes of $10 \% \mathrm{O}_{2}$ breathing. In contrast with 30 minutes of breathing $10 \% \mathrm{O}_{2}$, pretreatment with RS-120895 prevented the degranulation of MCs and the leukocyte-endothelial adherence of alveolar hypoxia. Numbers below photographs represent mean \pm SE of 5 rats in each group. LEA leukocyte-endothelial adherence, leukocytes $/ 100 \mu \mathrm{m}, \mathrm{MCDI}$, mast cell degranulation intensity, arbitrary units. ${ }^{*} P<0.01$ versus corresponding normoxic control rats. A complete set of the data for this series of experiments is included in the supplement.

The major source of the initial increase in plasma MCP-1 is evidently AM $\varnothing$. First, the reduction of $\mathrm{PO}_{2}$ induces a rapid release of MCP-1 from primary cultures of AM $\varnothing$ (Table 1). The effectiveness of AM $\varnothing$-borne MCP-1 in inducing MC degranulation is supported by the inhibitory effect of RS-102985 on the degranulation of MCs immersed in hypoxic AM $\varnothing$ supernatant (Table 2). Second, the depletion of AM $\varnothing$ by administration of clodronate liposomes prevented the increase in plasma MCP-1 observed during the first 30 minutes of hypoxia in conscious rats. At 60 minutes, plasma MCP-1 in AM $\varnothing$-depleted rats had increased to less than $50 \%$ of the value observed in the corresponding sample of intact animals (Figure 1A). The small number of AM $\varnothing$ remaining after 4 days of clodronate liposome treatment $(<5 \%$ of the number recovered by BAL from rats

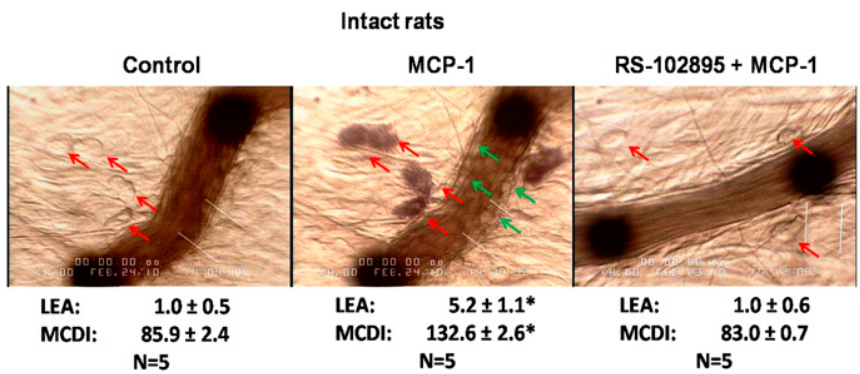

Figure 4. Left: Mesenteric postcapillary venule immediately before and 30 minutes after topical application of MCP-1 $(30 \mathrm{ng} / \mathrm{ml}$ dissolved in serum-free DMEM). Red arrows, MCs; green arrows, adherent leukocytes. Right: Mesenteric venule 30 minutes after application of MCP-1 in a rat pretreated with RS-102895 $(10 \mu \mathrm{M})$. Data represent the mean \pm SE of 5 rats per group. ${ }^{*} P<0.01$ versus corresponding control rats. A complete set of data for this series of experiments is included in the supplement.



Figure 5. Left: Postcapillary mesenteric venule of an AM $\varnothing$-depleted rat 30 minutes after topical application of $30 \mathrm{ng} / \mathrm{ml}$ of MCP-1 dissolved in serum-free DMEM. Right: Postcapillary mesenteric venule of an AM $\varnothing$ depleted rat 30 minutes after topical application of $30 \mathrm{ng} / \mathrm{ml}$ of MCP-1 dissolved in plasma from intact rats. Data represent the mean \pm SE of 5 rats in each group ${ }^{*} P<0.01$ versus corresponding control rats. A complete set of data for this series of experiments is included in the supplement.

treated with PBS liposomes) is unlikely to be responsible for this increase. Aside from the disproportionate concentration of plasma MCP-1 in relation to the number of AM $\varnothing$, the MCP-1 released from these remaining $\mathrm{AM} \varnothing$ would be expected to follow a time course similar to that observed in intact rats. Cells other than AM $\varnothing$ are probably the main contributors to the late increase in plasma MCP-1 in this case (30). Whether the stimulus for the delayed release of MCP-1 involves the reduced tissue $\mathrm{PO}_{2}$ or some other factor associated with hypoxia in the intact animals is not known. Nevertheless, although MCP-1 from these alternative sources may contribute to systemic inflammation at later times, the time course indicates that it does not play a role in the rapid onset of inflammation. Accordingly, these results indicate that AM $\varnothing$ is the predominant source of the early increase in plasma MCP-1 during hypoxia.

The putative circulating mediator of inflammation must also meet a second criterion, that is, its plasma concentration during hypoxia must be sufficient to activate MCs. The immersion of MCs in plasma samples drawn from intact, conscious rats breathing $10 \% \mathrm{O}_{2}$, in which elevated MCP-1 concentrations were documented (Figure 1A), produced substantial degranulation (Figure 1B). No degranulation was observed when MCs were immersed in the normoxic plasma of intact or AM $\varnothing$ depleted rats, or in the plasma of hypoxic, AM $\varnothing$-depleted rats (Figure 1B). The addition of MCP-1 to normoxic plasma or normoxic AM $\varnothing$ supernatants produced a concentrationdependent degranulation of immersed MCs (Figure 2). The percentage of degranulated MCs immersed in plasma samples drawn from intact rats breathing $10 \% \mathrm{O}_{2}$ (Figure $1 \mathrm{~B}$ ) was close to that seen when MCs were immersed in normoxic plasma or normoxic AM $\varnothing$ supernatants with similar MCP-1 concentrations (Figure 2). These results indicate that the plasma MCP-1 level reached by hypoxic rats with a normal AM $\varnothing$ count is sufficient to induce the degranulation of MCs, and suggest that the degranulation of MCs occurred as a result of the elevated MCP-1 concentration, and was not the result of other factors associated with hypoxia.

The participation of MCP-1 in the inflammation induced by alveolar hypoxia in intact animals was investigated using complementary approaches. On the one hand, pretreatment of the mesentery with RS-102985 prevented the rapid mesenteric 
microvascular effects observed in intact rats breathing $10 \% \mathrm{O}_{2}$ (Figure 3). RS-102895 is a selective antagonist of CCR2b, the putative MCP-1 receptor expressed in MC (31) and in endothelial cell membranes (32). Second, a topical application of MCP-1 into the mesenteric microcirculation of normoxic, intact rats replicated the inflammatory response to alveolar hypoxia (Figure 4). This response was prevented by an administration of RS-102985 in the same dose used to block the effects of alveolar hypoxia (Figure 3). Both RS-102985 and MCP-1 were administered topically. This method avoided potential systemic responses to these agents that could influence the results. The MCP-1 concentration of the solutions applied approximated the plasma MCP-1 concentration observed in intact rats during hypoxia.

In conclusion, a combination of in vivo and in vitro data show that AM $\varnothing$ release MCP-1 under hypoxia, and this release leads to an increase in plasma concentration of MCP-1, with a time course compatible with that of systemic inflammation. Furthermore, the plasma MCP-1 concentration attained at the onset of hypoxia was sufficient to induce a substantial degranulation of MCs, the first step in the systemic inflammatory cascade. Finally, the participation of MCP-1 in the systemic inflammation of hypoxia is supported by the attenuation of the mesenteric inflammatory markers by $\mathrm{CCR} 2 \mathrm{~b}$ receptor antagonists in rats breathing $10 \% \mathrm{O}_{2}$. This was complemented by the observation that the administration of MCP-1 to normoxic rats replicates the mesenteric response to alveolar hypoxia.

\section{Evidence Supporting the Existence of a MCP-1 Cofactor}

Although these results provide substantial evidence supporting MCP-1 as the AM $\varnothing$-borne circulating mediator of inflammation, MCP-1 also apparently requires a cofactor to activate MCs at concentrations observed under physiologically relevant conditions.

This finding was initially suggested by the observation that MCP-1 did not produce the degranulation of MCs immersed in normoxic cell culture medium, even at concentrations that were effective in plasma or in AM $\varnothing$ supernatants (Figure 2). This finding is consistent with earlier reports of a lack of effect of MCP-1 on the degranulation of in vitro MC cultures $(33,34)$. In fact, the release of histamine by rat peritoneal MC cultures was evident only when MCP-1 was administered in concentrations 10-20-fold greater than those seen in the plasma of hypoxic rats. Lower concentrations exerted no effect (35). These results were in marked contrast with the documented effectiveness of MCP-1 in activating MCs in vivo (26).

The filtration experiments provided more direct evidence for the existence of such a cofactor in normoxic plasma and AM $\varnothing$ supernatant. The degranulation of MCs induced by MCP-1 was interpreted as an indicator of the presence of the cofactor in a given fluid fraction. The results, which were essentially the same in plasma from intact normoxic rats and in normoxic AMØ supernatants, suggest that the cofactor is composed of at least two fractions: one with a molecular weight greater than $30 \mathrm{kD}$ and less than $50 \mathrm{kD}$, and the other with a molecular weight greater than $50 \mathrm{kD}$ and less than $100 \mathrm{kD}$ (Table 3).

Several lines of evidence suggest that the cofactor is constitutively secreted by AM $\varnothing$ and is normally present in plasma and extracellular fluid. First, AM $\varnothing$ are necessary for MCP-1 to induce the degranulation of MCs. MCs immersed in plasma obtained from AM $\varnothing$-depleted rats after 60 minutes of $10 \% \mathrm{O}_{2}$ breathing did not degranulate. This was the result despite the MCP-1 concentration of this sample, which was sufficient to produce a significant degranulation of MCs immersed in plasma from intact rats or in AM $\varnothing$ supernatants (Figure 2). These results are consistent with the results of filtration experiments that showed no MCP-1-induced degranulation of MCs in any of the fractions of plasma from AM $\varnothing$-depleted rats. Further support for the idea of an AM $\varnothing$-borne cofactor for MCP-1 is provided by the results depicted in Figures 4 and 5. Topical MCP-1 dissolved in serum-free DMEM replicated the response to hypoxia in the mesentery of intact, normoxic rats (Figure 4), whereas it had no effect on AMØ-depleted rats (Figure 5). On the other hand, MCP-1 dissolved in the plasma of rats with a normal AM $\varnothing$ count did elicit the degranulation of MCs and leukocyte-endothelial adherence in AM $\varnothing$-depleted rats. These results imply that the absence of the cofactor in the mesenteric environment of AM $\varnothing$-depleted rats prevented the degranulation of MCs when MCP-1 was dissolved in DMEM. However, when the cofactor was applied, using the plasma of rats with a normal AM $\varnothing$ count as a vehicle, MCP-1 did elicit the degranulation of MCs in the mesentery of $A M \emptyset$-depleted rats.

These results provide persuasive evidence for an AM $\varnothing$-borne cofactor of MCP-1. Its nature, however, remains unknown. Because this substance appears to be a normal component of body fluids that is not expressed exclusively under conditions of hypoxia, efforts directed toward its identification are likely to be complex and extensive, and as such, fall outside the scope of the present study. This research addressed the central question of whether MCP-1 is the AMØ-borne mediator of the systemic inflammation of hypoxia. The data strongly support this hypothesis. Within this context, the results indicate that a cofactor normally present in biological fluids is necessary for physiologically relevant concentrations of MCP- 1 to activate MCs. This issue should be considered when analyzing in vitro experiments involving MCP-1, when this cofactor may not be present.

\section{Functional Relevance of These Findings}

The present and previous studies $(12,16)$ highlight the systemic effects of activating AM $\varnothing$. In contrast with their better-known intrapulmonary effects, the extrapulmonary consequences of activating AM $\varnothing$ have attracted less attention. Recent, accumulating evidence points to a systemic inflammatory effect of the activation of AM $\varnothing$, secondary to the phagocytosis of inhaled particulate matter. Inflammatory mediators released by AM $\varnothing$ enter the circulation to produce inflammation and endothelial dysfunction in systemic microvascular beds (36-40). These AM $\varnothing$-mediated responses were proposed to underlie the elevated cardiovascular morbidity associated with environmental pollution (40). The phenomenon described in this research represents an example of the systemic response to AM $\varnothing$ activated by another stimulus (in this case, reduced alveolar $\mathrm{Po}_{2}$ ).

Clinical conditions as well as experimental interventions associated with alveolar hypoxia are accompanied by systemic effects, and in several of these, a systemic inflammatory component was identified. Examples include the sarcopenia and cardiovascular abnormalities of chronic obstructive pulmonary disease (1), the insufficient hemopoietic response in pulmonary fibrosis (2), and the cardiovascular and metabolic dysfunctions in sleep apnea (3). Under acute conditions, systemic responses with a possible inflammatory link include the multiple organ failure secondary to atelectasis (41), acute lung injury $(42,43)$ and pulmonary contusion $(44)$, the systemic inflammation of pneumonia (4), and the acute illnesses of high altitude (5). Inflammation may not play a causal role in every one of these examples. However, inflammation is likely to modify their development and outcome. The phenomenon described here could contribute to the initiation of the systemic inflammation observed in some of these conditions. In this respect, our studies of intact animals entailed a fairly severe degree of hypoxia: alveolar $\mathrm{PO}_{2}$ values in rats breathing $10 \% \mathrm{O}_{2}$ 
typically decrease to $45-50 \mathrm{~mm} \mathrm{Hg}$. This level of $\mathrm{PO}_{2}$ is evident, for instance, at an altitude of approximately 5,000 m, or under clinical conditions in which pulmonary function is seriously compromised. However, AM $\varnothing$ cultures released MCP-1 when exposed to a $\mathrm{PO}_{2}$ of approximately $70 \mathrm{~mm} \mathrm{Hg}$, and the response to this less severe decrease in $\mathrm{PO}_{2}$ was the same as that observed at lower $\mathrm{PO}_{2}$ values (Table 1). This finding would suggest that diseases presenting relatively moderate reductions in alveolar $\mathrm{PO}_{2}$ may elicit AM $\varnothing$-initiated systemic inflammation.

Alveolar hypoxia also produces an inflammatory response in the lungs, and AM $\varnothing$ play an important role in this response. Rats breathing $10 \% \mathrm{O}_{2}$ showed an extravasation of albumin and the expression of inflammatory cytokines $(45,46)$. These changes were attenuated by the selective depletion of AM $\varnothing$. In addition, hypoxia induces the expression of neurokinin-1 receptors in the lung, leading in turn to inflammatory responses mediated by IL-1, IL-6, and TNF- $\alpha$ (47). Whether similar mechanisms underlie the inflammatory responses of the lung and the systemic circulation to reduced alveolar $\mathrm{Po}_{2}$ should be the subject of future research.

Finally, we acknowledge that the exposure of an intact organism to hypoxia is a complex stimulus that elicits multiple responses. Mechanisms with a slower time course (in particular, the changes in gene expression initiated by a reduction in $\mathrm{Po}_{2}$ ) are likely to modify the effects of this initial response at later times in the course of hypoxia.

In conclusion, the present study provides substantial evidence to support the idea that the systemic inflammatory response to alveolar hypoxia is not initiated by a reduction of $\mathrm{PO}_{2}$ in the systemic microcirculation, but rather by the activation of MCs by a mediator released by AM $\varnothing$ and transported by the circulation. These results strongly support MCP-1 as this mediator.

Author Disclosure: None of the authors has a financial relationship with a commercial entity that has an interest in the subject of this manuscript.

\section{References}

1. Agusti A, Soriano JB. COPD as a systemic disease. COPD 2008;5:133138.

2. Tsantes A, Tassiopoulos S, Papadhimitriou SI, Bonovas S, Kavalierou L Vaiopoulos G, Meletis I. Suboptimal erythropoietic response to hypoxemia in idiopathic pulmonary fibrosis. Chest 2003;124:548-553

3. Jelic S, Padeletti M, Kawut SM, Higgins C, Canfield SM, Onat D Colombo PC, Basner RC, Factor P, LeJemtel TH. Inflammation, oxidative stress, and repair capacity of the vascular endothelium in obstructive sleep apnea. Circulation 2008;117:2270-2278.

4. Fernandez-Serrano S, Dorca J, Coromines M, Carratala J, Gudiol F, Manresa F. Molecular inflammatory responses measured in blood of patients with severe community-acquired pneumonia. Clin Diagn Lab Immunol 2003;10:813-820.

5. Basnyat B, Murdoch DR. High-altitude illness. Lancet 2003;361:19671974

6. Wood JG, Mattioli LF, Gonzalez NC. Hypoxia causes leukocyte adherence to mesenteric venules in nonacclimatized, but not in acclimatized, rats. J Appl Physiol 1999;87:873-881.

7. Dix R, Orth T, Allen J, Wood JG, Gonzalez NC. Activation of mast cells by systemic hypoxia, but not by local hypoxia, mediates increased leukocyte-endothelial adherence in cremaster venules. J Appl Physiol 2003;95:2495-2502.

8. Shah S, Allen J, Wood JG, Gonzalez NC. Dissociation between skeletal muscle microvascular $\mathrm{PO}_{2}$ and hypoxia-induced microvascular inflammation. J Appl Physiol 2003;94:2323-2329.

9. Mc Donald J, Wood J. Mast cell degranulation promotes the cerebral microvascular inflammatory response to hypoxia. FASEB $J$ 2003;17: A1282.

10. Wood JG, Johnson JS, Mattioli LF, Gonzalez NC. Systemic hypoxia promotes leukocyte-endothelial adherence via reactive oxidant generation. J Appl Physiol 1999;87:1734-1740.

11. Wood JG, Johnson JS, Mattioli LF, Gonzalez NC. Systemic hypoxia increases leukocyte emigration and vascular permeability in conscious rats. J Appl Physiol 2000;89:1561-1568.
12. Gonzalez NC, Allen J, Blanco VG, Schmidt EJ, van Rooijen N, Wood JG. Alveolar macrophages are necessary for the systemic inflammation of acute alveolar hypoxia. J Appl Physiol 2007;103:1386-1394.

13. Gonzalez NC, Allen J, Schmidt EJ, Casillan AJ, Orth T, Wood JG. Role of the renin-angiotensin system in the systemic microvascular inflammation of alveolar hypoxia. Am J Physiol Heart Circ Physiol 2007;292:H2285-H2294.

14. Steiner DR, Gonzalez NC, Wood JG. Mast cells mediate the microvascular inflammatory response to systemic hypoxia. J Appl Physiol 2003;94:325-334.

15. Kubes P, Granger DN. Leukocyte-endothelial cell interactions evoked by mast cells. Cardiovasc Res 1996;32:699-708.

16. Chao J, Wood JG, Blanco VG, Gonzalez NC. The systemic inflammation of alveolar hypoxia is initiated by alveolar macrophage-borne mediator(s). Am J Respir Cell Mol Biol 2009;41:573-582.

17. Chao J, Wood JG, Gonzalez NC. Alveolar hypoxia, alveolar macrophages, and systemic inflammation. Respir Res 2009;10:54.

18. Steiner DR, Gonzalez NC, Wood JG. Leukotriene B(4) promotes reactive oxidant generation and leukocyte adherence during acute hypoxia. J Appl Physiol 2001;91:1160-1167.

19. Orth T, Allen JA, Wood JG, Gonzalez NC. Plasma from conscious hypoxic rats stimulates leukocyte-endothelial interactions in normoxic cremaster venules. J Appl Physiol 2005;99:290-297.

20. Naidu BV, Krishnadasan B, Byrne K, Farr AL, Rosengart M, Verrier ED, Mulligan MS. Regulation of chemokine expression by cyclosporine $\mathrm{A}$ in alveolar macrophages exposed to hypoxia and reoxygenation. Ann Thorac Surg 2002;74:899-905, discussion 905.

21. VanOtteren GM, Standiford TJ, Kunkel SL, Danforth JM, Strieter RM. Alterations of ambient oxygen tension modulate the expression of tumor necrosis factor and macrophage inflammatory protein-1 alpha from murine alveolar macrophages. Am J Respir Cell Mol Biol 1995;13:399-409.

22. Ben-Baruch A, Michiel DF, Oppenheim JJ. Signals and receptors involved in recruitment of inflammatory cells. J Biol Chem 1995; 270:11703-11706.

23. Murphy PM. Chemokine receptors: structure, function and role in microbial pathogenesis. Cytokine Growth Factor Rev 1996;7:47-64.

24. Fuentes ME, Durham SK, Swerdel MR, Lewin AC, Barton DS, Megill JR, Bravo R, Lira SA. Controlled recruitment of monocytes and macrophages to specific organs through transgenic expression of monocyte chemoattractant protein-1. J Immunol 1995;155:5769-5776.

25. Carr MW, Roth SJ, Luther E, Rose SS, Springer TA. Monocyte chemoattractant protein 1 acts as a T-lymphocyte chemoattractant. Proc Natl Acad Sci USA 1994;91:3652-3656.

26. Wan MX, Wang Y, Liu Q, Schramm R, Thorlacius H. CC chemokines induce $\mathrm{P}$-selectin-dependent neutrophil rolling and recruitment in vivo: intermediary role of mast cells. Br J Pharmacol 2003;138:698-706.

27. Van Rooijen N, Sanders A. Liposome mediated depletion of macrophages: mechanism of action, preparation of liposomes and applications. J Immunol Methods 1994;174:83-93.

28. Futagami S, Hiratsuka T, Shindo T, Hamamoto T, Tatsuguchi A, Nobue U, Shinji Y, Suzuki K, Kusunoki M, Tanaka S, et al. COX-2 and CCR2 induced by CD40 ligand and MCP-1 are linked to VEGF production in endothelial cells. Prostaglandins Leukot Essent Fatty Acids 2008;78:137-146.

29. Metcalfe DD, Baram D, Mekori YA. Mast cells. Physiol Rev 1997;77: 1033-1079.

30. Sharma AK, Fernandez LG, Awad AS, Kron IL, Laubach VE. Proinflammatory response of alveolar epithelial cells is enhanced by alveolar macrophage-produced TNF-alpha during pulmonary ischemia-reperfusion injury. Am J Physiol Lung Cell Mol Physiol 2007;293:L105-L113.

31. Ko J, Yun CY, Lee JS, Kim DH, Yuk JE, Kim IS. Differential regulation of $\mathrm{CC}$ chemokine receptors by 9-cis retinoic acid in the human mast cell line, HMC-1. Life Sci 2006;79:1293-1300.

32. Salcedo R, Ponce ML, Young HA, Wasserman K, Ward JM, Kleinman HK, Oppenheim JJ, Murphy WJ. Human endothelial cells express $\mathrm{CCR} 2$ and respond to $\mathrm{MCP}-1$ : direct role of $\mathrm{MCP}-1$ in angiogenesis and tumor progression. Blood 2000;96:34-40.

33. Alam R, Forsythe PA, Stafford S, Lett-Brown MA, Grant JA. Macrophage inflammatory protein-1 alpha activates basophils and mast cells. J Exp Med 1992;176:781-786.

34. Fureder W, Agis H, Semper H, Keil F, Maier U, Muller MR, Czerwenka K, Hofler H, Lechner K, Valent P. Differential response of human basophils and mast cells to recombinant chemokines. Ann Hematol 1995;70:251-258.

35. Conti P, Boucher W, Letourneau R, Feliciani C, Reale M, Barbacane RC, Vlagopoulos P, Bruneau G, Thibault J, Theoharides TC. Mono- 
cyte chemotactic protein-1 provokes mast cell aggregation and [3H]5HT release. Immunology 1995;86:434-440.

36. Ishii H, Hayashi S, Hogg JC, Fujii T, Goto Y, Sakamoto N, Mukae H, Vincent R, van Eeden SF. Alveolar macrophage-epithelial cell interaction following exposure to atmospheric particles induces the release of mediators involved in monocyte mobilization and recruitment. Respir Res 2005;6:87.

37. Tamagawa E, Bai N, Morimoto K, Gray C, Mui T, Yatera K, Zhang X Xing L, Li Y, Laher I, et al. Particulate matter exposure induces persistent lung inflammation and endothelial dysfunction. Am J Physiol Lung Cell Mol Physiol 2008;295:L79-L85.

38. van Eeden SF, Tan WC, Suwa T, Mukae H, Terashima T, Fujii T, Qui D, Vincent R, Hogg JC. Cytokines involved in the systemic inflammatory response induced by exposure to particulate matter air pollutants (PM(10)). Am J Respir Crit Care Med 2001;164: $826-830$.

39. Nurkiewicz TR, Porter DW, Barger M, Millecchia L, Rao KM, Marvar PJ, Hubbs AF, Castranova V, Boegehold MA. Systemic microvascular dysfunction and inflammation after pulmonary particulate matter exposure. Environ Health Perspect 2006;114:412-419.

40. Simkhovich BZ, Kleinman MT, Kloner RA. Air pollution and cardiovascular injury epidemiology, toxicology, and mechanisms. J Am Coll Cardiol 2008;52:719-726.
41. Kisala JM, Ayala A, Stephan RN, Chaudry IH. A model of pulmonary atelectasis in rats: activation of alveolar macrophage and cytokine release. Am J Physiol 1993;264:R610-R614.

42. St. John RC, Mizer LA, Kindt GC, Weisbrode SE, Moore SA, Dorinsky PM. Acid aspiration-induced acute lung injury causes leukocytedependent systemic organ injury. J Appl Physiol 1993;74:1994-2003.

43. Puneet $\mathrm{P}$, Moochhala S, Bhatia M. Chemokines in acute respiratory distress syndrome. Am J Physiol Lung Cell Mol Physiol 2005;288:L3-L15.

44. Perl M, Gebhard F, Bruckner UB, Ayala A, Braumuller S, Buttner C, Kinzl L, Knoferl MW. Pulmonary contusion causes impairment of macrophage and lymphocyte immune functions and increases mortality associated with a subsequent septic challenge. Crit Care Med 2005;33:1351-1358.

45. Beck-Schimmer B, Schimmer RC, Madjdpour C, Bonvini JM, Pasch T, Ward PA. Hypoxia mediates increased neutrophil and macrophage adhesiveness to alveolar epithelial cells. Am J Respir Cell Mol Biol 2001:25:780-787.

46. Madjdpour C, Jewell UR, Kneller S, Ziegler U, Schwendener R, Booy C, Klausli L, Pasch T, Schimmer RC, Beck-Schimmer B. Decreased alveolar oxygen induces lung inflammation. Am J Physiol Lung Cell Mol Physiol 2003;284:L360-L367.

47. Zee ED, Schomberg S, Carpenter TC. Hypoxia upregulates lung microvascular neurokinin-1 receptor expression. Am J Physiol Lung Cell Mol Physiol 2006;291:L102-L110. 